\title{
Quantificação de capilares no tecido muscular esquelético em animais com insuficiência arterial periférica induzida submetidos a treinamento de endurance $^{1}$
}

\author{
Quantitative research into the skeletal muscle tissue of rats in endurance training \\ inducing neovascularization in the ischemic skeletal muscle
}

\section{Celina Cordeiro de Carvalho2, Sílvia Regina Arruda de Moraes3, Síntya Tertuliano Chalegre4, Tetsuo Tashiro5}

\author{
1. Trabalho realizado no Departamento de Biofísica e Radiologia da Universidade Federal de Pernambuco (UFPE). \\ 2. Profa. Mestre do Curso de Fisioterapia, Faculdade Integrada do Recife - Pernambuco. \\ 3. Prof ${ }^{a}$. Adjunto do Departamento de Anatomia da UFPE. \\ 4. Aluna do curso de Fisioterapia do Departamento de Biofísica da UFPE. \\ 5. Prof. Assistente do Departamento de Educação Física e do Desporte da UFPE.
}

\section{RESUMO}

OBJETIVO: Analisar quantitativamente o tecido muscular esquelético de ratos com insuficiência arterial periférica induzida após treinamento de endurance.

MÉTODOS: Os animais foram preparados cirurgicamente para a oclusão total da artéria femoral direita. Após o procedimento cirúrgico os ratos foram limitados a atividades na própria gaiola (sedentários, $n=10$ ) ou submetidos a treinamento de endurance com ciclo ergômetro de forma contínua (treinados, $n=10$ ), que consistia em caminhada 2x/dia (manhã e tarde) a 17 m/min, por 5 minutos, 5 dias/semana por 8 e 12 semanas. Os grupos foram subdivididos com seus respectivos grupos sedentários. A análise foi realizada pela observação histológica do músculo vasto medial direito após o período de teste.

RESULTADOS: No grupo de animais treinados durante 8 semanas, o número médio de capilares no tecido muscular foi maior $(5,2 \pm 3,834)$ do que no grupo sedentário $(0,6 \pm 0,894)$. No grupo de animais treinados durante 12 semanas o número médio de capilares foi maior $(6,8 \pm 3,033)$ do que no grupo sedentário $(3,0 \pm$ $2,345)$. O número médio de capilares entre os grupos treinados de 12 e 8 semanas também foi maior no primeiro, entretanto, não estatisticamente significante. O número médio de capilares do grupo sedentário de 12 semanas foi maior $(3,0 \pm 2,345)$ do que no grupo de 8 semanas $(0,6 \pm 0,894)$.

CONCLUSÃO: Em animais com insuficiência arterial periférica induzida submetidos a treinamento de endurance, há um processo de adaptação muscular com um aumento no número de capilares.

DESCRITORES: Neovascularização. Músculo esquelético. Treinamento de endurance.

\begin{abstract}
PURPOSE: This work introduces quantitative research into the skeletal muscle tissue of rats, suffering induced outlying arterial inadequacy after endurance training.

METHODS: All those rats had been prepared surgically towards the right femoral artery total occlusion.
\end{abstract} After surgical procedures, the rats were either restricted to activities inside their cage (sedentary ones, $n=10$ ) 
or undergone endurance training with cycle ergometer in continuous way (trained ones, $n=10$ ), which consisted in walking twice a day (morning and afternoon) at $17 \mathrm{~m} / \mathrm{min}$, for 5 minutes, 5 days a week during 8 and 12 weeks. The groups were halved in order with their respective sedentary groups. The analysis was achieved by vast medial muscle histological observation after test period.

RESULTS: Considering the eight-week trained group, the average number of muscle tissue capillary vessels has been shown higher $(5,2 \pm 3,834)$ than in the sedentary one $(0,6 \pm 0,894)$. Also in the 12 -week trained group, the average number of capillary vessels has been shown higher $(6,8 \pm 3,033)$ than in the sedentary one $(3,0 \pm 2,345)$. The average number of capillary vessels between the 12-week and 8-week groups has been shown higher in the first group as well, however, not statistically significant. The average number of the 12week sedentary group's capillary vessels has been higher $(3,0 \pm 2,345)$ than the eight-week one's $(0,6 \pm$ $0,894)$.

CONCLUSION: The muscle adaptive process concerning the capillary vessels' increasing number through the endurance training of rats, which have induced outlying arterial inadequacy.

KEY WORDS: Neovascularization. Skeletal muscle. Endurance training.

\section{Introdução}

O termo angiogênese foi introduzido por Hertig em 1935 para descrever a formação de novos vasos sangüíneos na placenta e, mais tarde, Folkman em 1971, para descrever a neovascularização acompanhando o crescimento de tumores sólidos ${ }^{1}$. O estudo da angiogênese começou há cerca de três décadas na tentativa de entender o papel da vascularização no crescimento tumoral. Ele é agora um vasto campo que inclui o crescimento e a regressão de capilares no desenvolvimento embrionário, nas funções fisiológicas, e nos estados patológicos².

Apesar de intensas investigações sobre a angiogênese, pouco esforço tem sido feito para explorar este fenômeno no tecido isquêmico. Os poucos estudos sobre este tópico tem focado primariamente o desenvolvimento de artérias colaterais em leitos arteriais existentes ${ }^{3}$.

Hudlická e col. ${ }^{4}$, em extensiva revisão dos numerosos fatores metabólicos, mecânicos, humoral e hormonal, envolvidos no desenvolvimento capilar e adaptações do músculo esquelético, sugeriram que a danificação da membrana basal como um resultado de tensão da parede do vaso, ou na liberação de enzimas lisossomais e proteases ácidas, são necessárias para anteceder a angiogênese.

Foi observado que ratos machos com ligadura de artéria femoral bilateral, treinados em programa de treinamento físico de média intensidade na esteira ergométrica (2x/dia, 15m/min, 5 dias/semana por 811 semanas), têm seus fluxos sangüíneos musculares dependentes de colaterais aumentados pelo treinamento. Isto foi coincidente com uma melhora na tolerância dos animais ao exercício durante a caminhada na esteira ${ }^{5}$.

Estudos sobre o fluxo sangüíneo muscular em ratos com insuficiência arterial periférica induzida demonstraram que ocorreu uma redistribuição do fluxo sangüíneo nos animais treinados, aumentando o fluxo sangüíneo aproximadamente em $80 \%$ nos tecidos proximais. $\mathrm{O}$ aumento associado ao aporte de $\mathrm{O}_{2}$ para o músculo relativamente isquêmico, provavelmente contribuiu para aumentar marcadamente a tolerância evidenciada nos tecidos treinados ${ }^{6}$. Isso ocorre principalmente em resposta à presença de fatores angiogênicos liberados por (1) tecidos isquêmicos, (2) tecidos que estão crescendo rapidamente, ou (3) tecidos que têm metabolismo excessivamente alto ${ }^{7}$. Porém, o passo essencial da angiogênese dependerá do estímulo angiogênico, diferenciação entre os tecidos e pode ser determinado pelo estado fisiopatológico. A sobrecarga muscular induz o crescimento capilar pelo brotamento lateral de capilares pré-existentes, provavelmente devido à ação mecânica do estiramento no lado luminal dos vasos. O mecanismo de formação do lume inclui a canalização inter e intracelular, enquanto que a mitose de células endoteliais, mas não a quebra da membrana basal, foi tida como o início do brotamento ${ }^{8}$.

A resposta da microcirculação a diferentes programas de treinamento tem sido observada por diferentes métodos com objetivos diferentes em humanos, bem como em músculos esqueléticos de 
animais. Um aumento na capilarização tem sido largamente descrito como um efeito específico do exercício de endurance no tecido muscular ${ }^{9}$.

O treinamento induzido aumenta a capacidade oxidativa no músculo esquelético e reflete o padrão de fibras musculares recrutadas durante o exercício. Foi previamente reportado que treinamento de endurance aumenta a capacidade do leito vascular no músculo esquelético, para transportar substâncias para o músculo e do músculo. Aumentos na capacidade do fluxo sangüíneo foi mais evidente em tecidos musculares com o melhor aumento relativo na atividade durante o treinamento. Por exemplo, treinamentos de baixa intensidade aumentam a capacidade nas fibras vermelhas oxidativas predominantemente dos membros inferiores, no entanto treinamentos de alta intensidade aumentam a capacidade do fluxo nas fibras brancas glicolíticas predominantemente dos músculos dos membros inferiores. Esses resultados foram conduzidos a propostas que treinamentos de diferentes intensidades produzem mudanças no leito vascular dos músculos que tem um melhor aumento relativo na atividade durante o treinamento ${ }^{10}$.

O presente estudo tem como objetivo verificar os efeitos do treinamento de endurance sobre o número de capilares no tecido muscular esquelético na insuficiência arterial periférica induzida.

\section{Métodos}

O estudo foi aprovado pela Comissão de Ética em Experimentação Animal do Centro de Ciências Biológicas da Universidade Federal de Pernambuco (CEEA-UFPE), processo No 013027/2003-35 e encontra-se de acordo com as normas vigentes.

Foram utilizados 20 ratos Wistar, machos, adultos jovens, pesando cerca de $250 \mathrm{~g}$. Os animais foram mantidos em gaiolas coletivas (no máximo 5 animais/ gaiola) com oferta de água e alimento Labina Purina ${ }^{a}$ ad libitum, sendo mantido um ciclo claro/escuro de 12/12 horas em temperatura ambiente.

Os animais foram separados aleatoriamente em grupo de animais treinados $(n=10)$ e o grupo de animais sedentários $(n=10)$ constituído por animais não submetidos ao treinamento. O grupo de animais treinados foi submetido à caminhada em um ciclo ergômetro, desenvolvido pelo Departamento de Mecânica do CEFET - PE, com uma velocidade de
$17 \mathrm{~m} / \mathrm{min}$, por cinco minutos, duas vezes ao dia e por 10 dias, para se familiarizar com o protocolo segundo Yang ${ }^{5}$, modificado. Os demais se limitaram a atividades dentro da própria gaiola.

No décimo primeiro dia, os dois grupos de animais foram preparados cirurgicamente para a ligadura da artéria femoral direita. Após depilação e anti-sepsia da região interna da pata traseira direita, foram anestesiados com uma solução de cloridrato de xilazina e quetamina, em uma quantidade de 0,2 ml para cada $100 \mathrm{~g}$ de peso. A via de aplicação foi a intramuscular, na região interna da pata traseira esquerda do animal. Após a incisão na face medial da pata traseira direita do animal de aproximadamente $3 \mathrm{~cm}$ a partir do ligamento inguinal, foi dissecada a artéria femoral direita em sua porção mais proximal (5-6 mm do ligamento inguinal). A ligadura foi feita com linha de algodão $n^{\circ} 50$ ao redor da artéria femoral, até sua total oclusão. A sutura da pele foi feita através de três pontos duplos com fio de sutura categut simples número 4-0. Ao final do procedimento foi administrado antibiótico tópico de rivamicina, sendo a mesma aplicação mantida diariamente na ferida operatória durante três dias.

Após três dias do procedimento cirúrgico, os animais do grupo treinado foram submetidos ao treinamento de endurance contínuo em ciclo ergômetro, duas vezes ao dia (manhã e tarde), 5 dias por semana, caminhando a uma velocidade de $17 \mathrm{~m} /$ min, por 5 minutos. O treinamento de endurance foi realizado de forma contínua, sendo, desta forma, mantida tanto a velocidade $(17 \mathrm{~m} / \mathrm{min})$ quanto o tempo de treinamento (5 minutos). O grupo dos animais treinados foi dividido em dois subgrupos: grupo treinado durante oito semanas ( $n=5)$; e o grupo treinado durante 12 semanas $(n=5)$. Os animais do grupo sedentário também foram subdivididos, ambos contendo cinco animais.

O primeiro grupo de animais (8 semanas de treinamento), foi sacrificado no dia imediatamente após o término do treinamento e o segundo (12 semanas de treinamento) com mais quatro semanas, após o término do treinamento. Sendo o mesmo número correspondente de animais para os grupos sedentários, respectivamente, e escolhidos de forma aleatória para a realização do sacrifício. O sacrifício foi realizado com uma dosagem de inalação letal de éter sulfúrico após serem anestesiados com uma solução de cloridrato de xilazina e quetamina por via 
intramuscular, em uma quantidade de 0,2 ml para cada $100 \mathrm{~g}$ de peso. O músculo vasto medial direito de todos os animais foi dissecado e em seguida seccionado transversalmente $(5 \mathrm{~mm})$ imediatamente após a morte do animal. As amostras foram colocadas em um recipiente de vidro com rolha contendo solução fixadora Bouin (75 ml de solução aquosa de ácido pícrico, $20 \mathrm{ml}$ de formaldeído a $40 \%$ e $5 \mathrm{ml}$ de ácido acético glacial) e após 24 horas, trocada por uma solução de álcool a 70\% ficando este até o processamento histológico do material, cujos cortes foram tratados com técnica de rotina histológica e corados com hematoxilina e eosina.

O material obtido foi analisado através de microscopia óptica, num microscópio Leica ${ }^{\circledR}$ modelo DMLS, utilizando-se um aumento de 400x. A análise quantitativa foi realizada comparativamente entre cada grupo (8 e 12 semanas) e entre os grupos de animais sedentários e os que foram submetidos ao treinamento. Os dados obtidos foram analisados pelo teste Mann-Witney com significância 0,05 e os resultados expressos em média \pm desvio padrão. Foi utilizado o programa Excel 2000 Microsoft $^{\circledR}$ para a montagem dos bancos de dados e gráficos.

\section{Resultados}

Após o programa de treinamento de endurance durante oito semanas em animais com insuficiência arterial periférica de membro inferior induzida cirurgicamente, o número médio de capilares no tecido muscular esquelético (Figura 1 ) foi maior no grupo treinado $(5,2 \pm 3,834, p<0,05)$ do que no grupo sedentário $(0,6 \pm 0,894, p<0,05)$ como ilustrado na Figura 2A. No grupo de animais treinados durante 12 semanas, o número médio de capilares no tecido muscular esquelético foi, também maior no grupo treinado $(6,8 \pm 3,033, p<0,05)$, apesar da relação
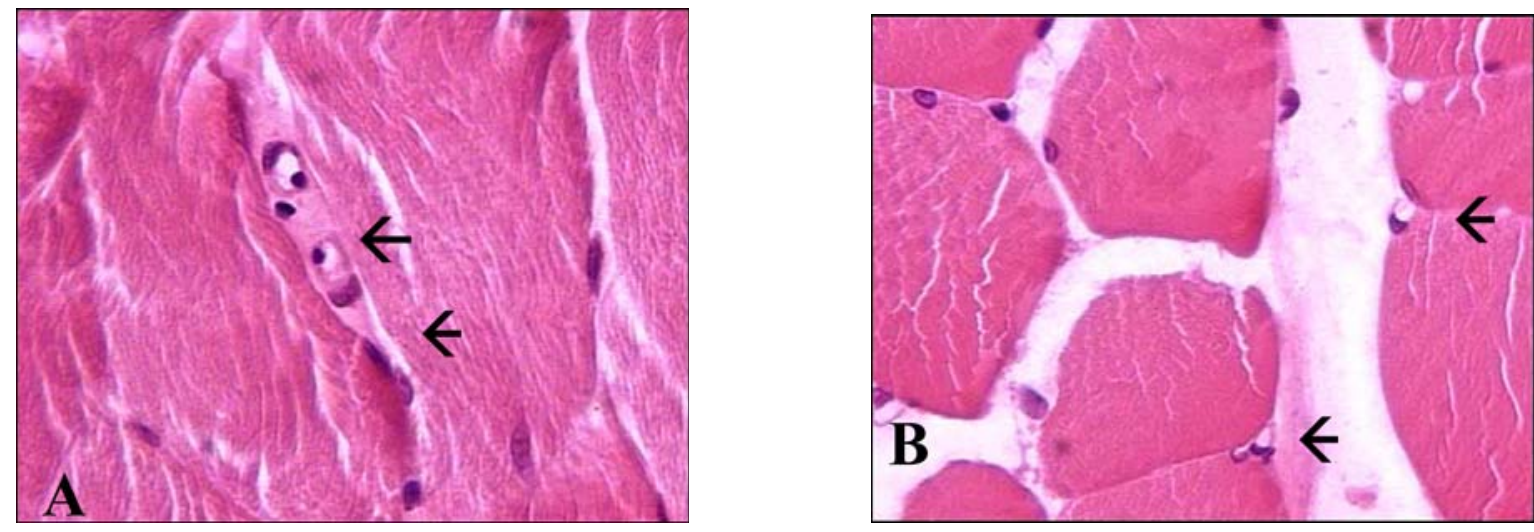

FIGURA 1 - Fotomicrografias do músculo vasto medial direito. A e B - Cortes transversais da porção mais proximal à oclusão da artéria femoral direita mostrando as células endoteliais dos capilares (setas) entre os feixes musculares. Hematoxilina e eosina - 4000x.

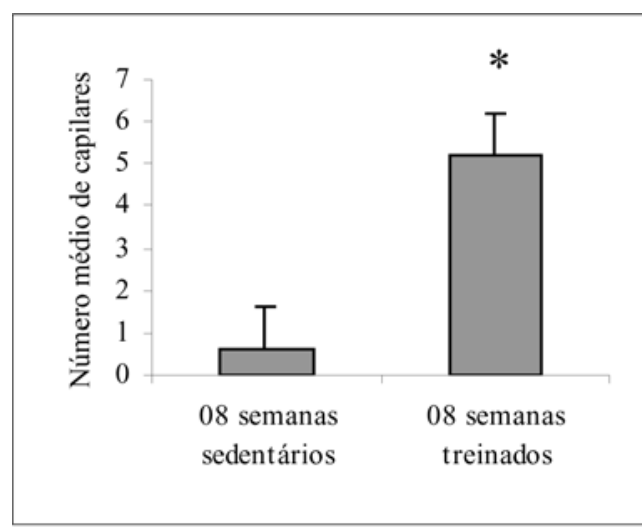

A

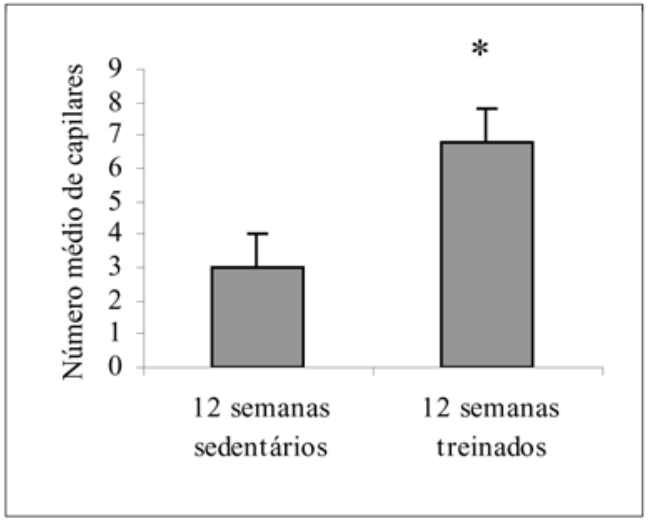

B

FIGURA 2 - Valores médios do número de capilares no músculo vasto medial direito animais sedentários e submetidos ao treinamento de endurance. A. durante 8 semanas após a ligadura da artéria femoral direita; B. durante 12 semanas após a ligadura da artéria femoral direita. *Relação estatisticamente significante $(\mathrm{P}<0,05$, para o Teste de Mann-Whitney). 
entre os animais treinados e o seu grupo sedentário respectivo ser menor, comparando-se com a mesma relação do grupo de animais com 8 semanas, o número médio de capilares encontrados neste grupo de animais treinados pelo período em questão, foi maior que no grupo sedentário (3,0 $\pm 2,345, p<$ 0,05) (Figura 2B).

O número médio de capilares no tecido muscular esquelético do grupo treinado durante 12 semanas foi maior $(6,8 \pm 3,033)$ do que o número médio de capilares no grupo treinado durante 8 semanas $(5,2$ $\pm 3,834$ ), porém não houve diferença estatisticamente significante $(p>0,05)$ (Figura 3A). O número médio de capilares no tecido muscular esquelético do grupo sedentário de 12 semanas foi maior (3,0 $\pm 2,345$, $p$ $<0,05)$ do que o número médio de capilares do grupo sedentário de 8 semanas $(0,6 \pm 0,894, p<0,05)$ (Figura 3B).

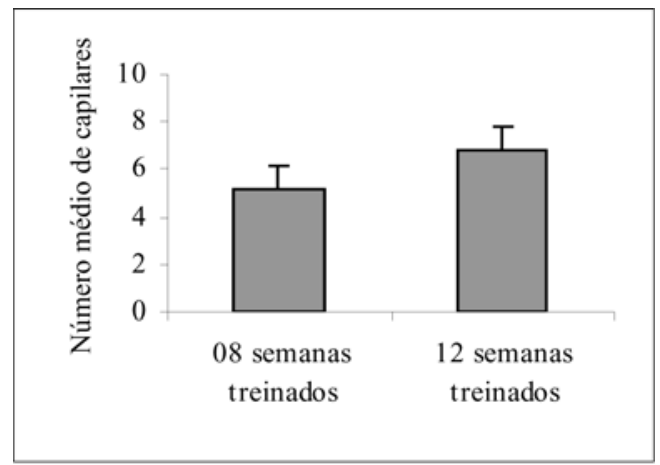

A

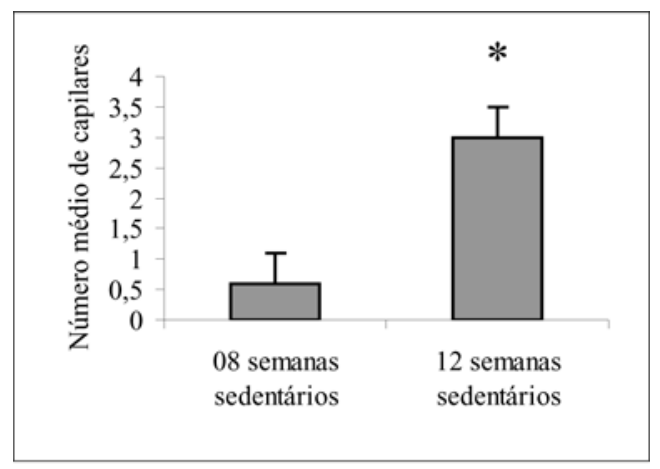

B

FIGURA 3 - Valores médios do número de capilares no músculo vasto medial direito. A. animais submetidos ao treinamento de endurance durante 8 e 12 semanas após a ligadura da artéria femoral direita; B. animais sedentários durante 8 semanas após a ligadura da artéria femoral direita. *Relação estatisticamente significante $(\mathrm{P}<0,05$, para 0 Teste de Mann-Whitney).

\section{Discussão}

Os resultados obtidos no modelo experimental que utilizamos para analisar o número de capilares no tecido muscular esquelético em animais com insuficiência arterial periférica induzida submetidos a treinamento de endurance são compatíveis com os descritos na literatura utilizando a esteira ergométrica ${ }^{3,8,9,11,12,13,14}$. Este modelo mostrou-se adequado para o desenvolvimento de novos capilares utilizando o ciclo ergômetro ${ }^{11,15}$.

A partir da $8^{a}$ semana de treinamento de endurance, foi observado um aumento no número de capilares que se estendeu até as 12 semanas de treinamento, apesar de ser verificado um aumento no número médio de capilares em relação aos animais analisados com 8 semanas de treinamento, esta diferença não foi estatisticamente significante ( $p>$ $0,05)$. Estes resultados sugerem que um treinamento de endurance durante 8 semanas em animais com insuficiência vascular periférica, induz a adaptações vasculares que melhoram o fluxo sangüíneo muscular durante o exercício, apesar de Suzuki et al ${ }^{16}$ terem mostrado processo semelhante observado com um treinamento de apenas seis semanas.

A quantidade de capilares encontrados na amostra do músculo dos animais treinados por $8 \mathrm{e}$ 12 semanas foi significantemente maior $(p<0,05)$ em comparação com os animais de seus respectivos grupos sedentários. Em geral, o treinamento de endurance induz um aumento da capilarização, o qual é precedido por um aumento da capacidade oxidativa que pode ser avaliada pelo crescimento da atividade enzimática oxidativa e pelo aumento da densidade de volume mitocondrial. $\mathrm{O}$ aumento da densidade capilar está, entretanto, algumas vezes ligado à diminuição do diâmetro da fibra muscular, porém, Hermansen e Wachtlová em 1971, observaram aumento da densidade capilar em treinamento de endurance associado às condições de hipertrofia das fibras musculares ${ }^{4,17}$.

O músculo esquelético não é homogêneo mas composto de diferentes tipos de fibras musculares e cada uma das quais possui um fenótipo próprio. O aumento da capilarização em treinamentos de endurance estava usualmente ligado às mudanças na composição das fibras musculares, que passam de fibras glicolíticas rápidas para fibras oxidativas. Foi demonstrado que a densidade capilar aumentaria 
mesmo em condições em que as fibras musculares não se alteram, indicando verdadeiro crescimento capilar, porém esse efeito seria maior ao redor de fibras oxidativas rápidas e lentas do que em regiões próximas às glicolíticas ${ }^{4,10}$.

A escolha de animais adultos jovens foi estabelecida em decorrência dos animais, nesse período, estarem na fase adulta e as modificações e respostas cardiovasculares se mostrarem mais estabilizadas. Isto então, foi considerado como parâmetro importante, visto que o crescimento de novos capilares não está geralmente presente em tecidos adultos normais, exceto no sistema reprodutor feminino devido à alta demanda de células endoteliais e no músculo esquelético por ser o surgimento de novos capilares bastante intenso durante o período de desenvolvimento pós-natal, e por atingir sua estabilidade na fase adulta ${ }^{8,16}$. No entanto, os mecanismos pelos quais a idade pode limitar a formação de novos vasos sangüíneos ainda permanecer bastante indefinida, apesar de Rivard et $\mathrm{al}^{13}$, confirmarem o prejuízo deste processo em idades avançadas devido à redução da capacidade de desenvolvimento de vasos colaterais em resposta a um estímulo angiogênico.

O procedimento cirúrgico de oclusão da artéria femoral direita, criou sinais agudos de insuficiência arterial periférica nos grupos em estudo. O fluxo reserva para o membro inferior direito foi reduzido. No entanto, a capacidade de fluxo colateral distal para este membro permaneceu suficiente para suprir as necessidades teciduais básicas dos grupamentos musculares, visto que após a oclusão aguda da artéria femoral, a região tecidual afetada estará completamente dependente da circulação colateral para seu suprimento de nutrientes ${ }^{14}$.

Assim, os animais que não foram submetidos a nenhum tipo de atividade que viesse a aumentar este fluxo para o membro insuficiente, não sofreram maiores necessidades de suprimento. Devido a isso, este procedimento não produziu sinais de isquemia no repouso e/ou necrose tecidual, nos animais sedentários e mesmo nos submetidos ao treinamento de endurance. Isto pode ser explicado pelo mecanismo fisiológico de resposta a estas necessidades metabólicas imediatas ${ }^{7}$.

A hipóxia é considerada um estímulo para o crescimento capilar, estimulando a liberação de fatores de crescimento derivados de macrófagos ${ }^{3,4}$.
Por isso é que, ao ser submetido a altas exigências metabólicas, como a uma atividade física crônica, estimula-se não só esses fatores de crescimento vascular $^{4,7,14,18}$ como também os fatores vasodilatadores, como o lactato e principalmente a adenosina $^{4,7}$.

Mas não somente através de fatores metabólicos e de crescimento vascular, é ativado o processo angiogênico. Os fatores mecânicos, induzidos pelo aumento do fluxo sangüíneo, também favorecem o crescimento capilar. Devido ao fluxo no músculo esquelético ser regulado por múltiplos mecanismos, é comum que esses fatores se tornem importantes moduladores na hiperemia induzida mecanicamente após o exercício físico ${ }^{19}$. A tensão provocando estresse na parede do vaso, pelo treinamento de endurance, levando a um aumento da pressão dentro dele, é, sem dúvida, indutora da proliferação vascular principalmente nos vasos de maior calibre ${ }^{4,7,8,14}$.

O desempenho na realização do exercício melhorou com o passar das sessões do programa de treinamento de endurance, possivelmente, por promover um aumento da capacidade aeróbica do músculo, aumentando o número e o volume de mitocôndrias, e a densidade capilar dos músculos ativos. Essas adaptações funcionais e estruturais ao nível dos capilares são importantes na estabilização da capacidade de fluxo e contribui para a regulação do fluxo sangüíneo nos músculos ativos durante o exercício. Elas também devem diminuir a distância de difusão entre capilares e mitocôndria ${ }^{14}$.

No treinamento de endurance durante 8 semanas em ratos com insuficiência arterial periférica induzida do membro inferior, o número de capilares aumentou significativamente $(p<0,05)$ quando comparado com o seu grupo de animais sedentário. Da mesma forma, um programa de treinamento de endurance em uma igual população de ratos submetidos a 12 semanas de treinamento, resultou em um aumento também significante $(p<0,05)$ no número de capilares em relação ao seu grupo de animais sedentário. Porém, se verificarmos a relação de cada grupo submetido ao treinamento de endurance e seu grupo sedentário, veremos que, entre os animais treinados durante 12 semanas, ela é menor que a dos animais treinados durante 8 semanas. Isto pode ser justificado não só pela adaptação muscular sofrida pelo treinamento a que foram submetidas ${ }^{3,4,7,14,16}$, mas, também, devido ao processo de acomodação cardiovascular sofrido 
pela manutenção, tanto da intensidade (17 m/min) quanto da duração da atividade física (5 minutos), imposta àquele grupamento muscular.

Os efeitos do treinamento são influenciados por muitos fatores, incluindo (1) a intensidade das sessões; (2) a freqüência e a duração do programa de treinamento; (3) o tipo de programa de treinamento; (4) a modalidade do exercício escolhida; e (5) a manutenção dos efeitos deste programa. Esses efeitos é que irão garantir aumentos máximos na aptidão $\left(\mathrm{VO}_{2}\right.$ máx) do sistema cardiovascular ${ }^{17}$.

Quando comparados os resultados do treinamento de endurance durante 8 e 12 semanas observou-se um aumento no número médio de capilares no grupo de 12 semanas, porém, estatisticamente não significante $(p>0,05)$. Já com relação aos resultados dos grupos de animais sedentários de 8 e 12 semanas, houve uma diferença significativa $(p<0,05)$, podendo isto ser explicado, pelo fato desses animais terem sofrido situações de hipóxia tecidual, em decorrência do processo oclusivo, que estimulou à liberação de fatores de crescimento e produção de substâncias vasodilatadoras no local da lesão e do estímulo o $^{3,4,7,14,16}$.

\section{Conclusões}

O estudo do número de capilares no tecido muscular em animais com insuficiência arterial periférica do membro inferior direito induzida cirurgicamente, sugere que, o treinamento de endurance durante oito semanas se mostra eficiente para promover um aumento do número médio de capilares no tecido muscular esquelético. A resposta tecidual nos grupos de animais sedentários ocorre desde o início do processo oclusivo, porém, se torna mais evidente após um período maior de recuperação, com 12 semanas.

\section{Referências}

1. Buschmann I, Schaper W. Arteriogenesis versus angiogenesis: two mechanisms of vessel growth. News Physiol Sci 1999; 14: 121-5.

2. Folkman J. Angiogenesis: what make blood vessels grow? News Physiol Sci 1986; 1:199-222.

3. Pevec WC, Ndoye A, Brinsky JL, Wiltse S, Cheung ATW. New blood vessels can be induced to invade ischemic skeletal muscle. J Vasc Surg 1996; 24: 534-44.

4. Hudlická O, Brown M, Egginton. Angiogenesis in skeletal and cardiac muscle. Physiol Rev 1992; 72: 369-417.
5. Yang HT, Ogilvie RW, Terjung RL. Training increases collateral-dependent muscle blood flow in aged rats. Am J Physiol 1995; 37: 1174-80.

6. Yang HT, Dinn RF, Terjung RL. Training increases muscle blood flow in rats with peripheral arterial insufficiency. J Appl Physiol 1990; 4: 1353-9.

7. Guyton AC. Tratado de fisiologia médica. 8ed. Rio de Janeiro: Guanabara Koogan; 1992.

8. Zhou A-L, Egginton S, Brown MD, Hudlická O. Capillary growth in overloaded, hypertrophic adult rat skeletal muscle: an ultra structural study. Anat Rec 1998; 252: 4963.

9. Soares JMC. Effects of training on muscle capillary pattern: intermittent vs continuous exercise. J Sports Med Phys Fitness 1992; 32: 123-7.

10. Sexton WL, Laughlin M. Harold. Influence of endurance exercise training on distribution of vascular adaptations in rat skeletal muscle. Am J Physiol 1994; 266: H483-90.

11. Fernhall BO, Kohrt W. The effect of training specificity on maximal and submaximal physiological responses to treadmill and cycle ergometry. J Sports Med Fitness 1990; 30: 268-75.

12. Yang HT, Ogilvie RW, Terjung RL. Training increases collateral-dependent muscle blood flow in aged rats. Am J Physiol 1995; 37: 1174-80.

13. Rivard A, Fabre J-E, Silver M. Age-dependent impairment of angiogenesis. Circulation 1999; 99: 111-20.

14. Yang HT, Laughlin MH, Terjung RL. Prior exercise training increases collateral-dependent blood flow in rats after acute femoral artery occlusion. Am J Physiol Heart Circ Physiol 2000; 279: H1890-7.

15. Hill PD. Treadmills and ergometers. Eur J Vasc Endovasc Surg 1998; 16: 449-51.

16. Suzuki J, Kobayashi T, Uruma T, Koyama T. Time-course changes in arteriolar and venular portions of capillary in Young treadmill-trained rats. Acta Physiol Scand 2001; 171: 77-86.

17. Fox EL, Bowers RW, Foss ML. Bases fisiológicas da Educação Física e dos Desportos. 4ed. Rio de Janeiro: Guanabara Koogan; 1991.

18. Folkman J. Therapeutic angiogenesis in ischemic limbs. Circulation 1998; 97: 1108-10.

19. Delp MD. Control of skeletal muscle perfusion at the onset of dynamic exercise. Med Sci Sports Exerc 1999; 31: 1011-8.

\section{Agradecimentos}

Nossos agradecimentos ao $\mathrm{CNPq}$, ao Departamento de Mecânica do CEFET-PE, ao Departamento de Anatomia da UFPE, e ao Programa de Pós-Graduação em Morfologia da UFPE. 
Correspondência:

Celina Cordeiro de Carvalho

Rua Rio Tejipió, 183/201

50721-640 Recife-PE

Tel: (81)3226-4459 / 9132-3733

celina@fir.br

Recebimento: 15/06/2004

Revisão: 20/07/2004

Aprovação: 18/08/2004

Conflito de interesse: nenhum

Fonte de financiamento: nenhuma

\section{Como citar este artigo:}

Carvalho CC, Moraes SRA, Chalegre ST, Tashiro T. Quantificação de capilares no tecido muscular esquelético em animais com insuficiência arterial periférica induzida submetidos a treinamento de endurance. Acta Cir Bras [serial online] 2004 SetOut;19(5). Disponível em URL: http://www.scielo.br/acb [também em CD-ROM].

*Figuras coloridas disponíveis em $\underline{w w w . s c i e l o . b r / a c b}$ 